\title{
Lenvatinib prolongs the progression-free survival time of patients with intermediate-stage hepatocellular carcinoma refractory to transarterial chemoembolization: A multicenter cohort study using data mining analysis
}

\author{
SHIGEO SHIMOSE ${ }^{1}$, TAKUMI KAWAGUCHI ${ }^{1}$, MASATOSHI TANAKA ${ }^{2}$, HIDEKI IWAMOTO $^{1}$, \\ KEN MIYAZAKI $^{1}$, ETSUKO MORIYAMA ${ }^{1}$, HIROYUKI SUZUKI ${ }^{1}$, TAKASHI NIIZEKI ${ }^{1}$, \\ TOMOTAKE SHIRONO ${ }^{1}$, MASAHITO NAKANO ${ }^{1}$, HIDEYA SUGA ${ }^{3}$, TAIZO YAMAGUCHI ${ }^{4}$, \\ YOSHINORI YOKOKURA ${ }^{2}$, KAZUNORI NOGUCHI $^{5}$, HIRONORI KOGA $^{1}$ and TAKUJI TORIMURA ${ }^{1}$
}

\author{
${ }^{1}$ Division of Gastroenterology, Department of Medicine, Kurume University School of Medicine, Kurume, Fukuoka 830-0011; \\ ${ }^{2}$ Department of Gastroenterology and Hepatology, Yokokura Hospital, Miyama, Fukuoka 839-0295; ${ }^{3}$ Department of \\ Gastroenterology and Hepatology, Yanagawa Hospital, Yanagawa, Fukuoka 832-0077; \\ ${ }^{4}$ Department ofGastroenterology and Hepatology, Iwamoto Internal Medical Clinic, Kitakyushu, Fukuoka 802-0832; \\ ${ }^{5}$ Department of Gastroenterology and Hepatology, Omuta City Hospital, Omuta, Fukuoka 836-8567, Japan
}

Received December 17, 2019; Accepted April 9, 2020

DOI: $10.3892 / 01.2020 .11758$

\begin{abstract}
Tyrosine kinase inhibitors are considered for use in patients with hepatocellular carcinoma (HCC) refractory to transarterial chemoembolization (TACE). The aim of the present retrospective study was to identify factors associated with progression-free survival (PFS) and to evaluate the indications for lenvatinib treatment in patients with intermediate-stage HCC refractory to TACE using a data-mining analysis. A total of 171 patients with intermediate-stage $\mathrm{HCC}$ refractory to TACE were included. All patients were classified into three groups according to their HCC treatment: Lenvatinib $(n=45)$, sorafenib $(n=53)$ and TACE $(n=73)$ groups. PFS time was calculated using the Kaplan-Meier method and analyzed using a log-rank test. Factors associated with PFS time were evaluated using multivariate and decision-tree
\end{abstract}

Correspondence to: Dr Shigeo Shimose, Division of Gastroenterology, Department of Medicine, Kurume University School of Medicine, 67 Asahi-machi, Kurume, Fukuoka 830-0011, Japan

E-mail: shimose_shigeo@med.kurume-u.ac.jp

Abbreviations: HCC, hepatocellular carcinoma; TACE, transarterial chemoembolization; TKI, tyrosine kinase inhibitor; OS, overall survival; PFS, progression-free survival; AEs, adverse events; ALBI, albumin-bilirubin; NLR, neutrophil to lymphocyte ratio; ORR, objective response rate; DCR, disease control rate; VEGF, vascular endothelial growth factor; FGF, fibroblast growth factor

Key words: hepatoma, tyrosine-kinase inhibitor, transarterial chemoembolization failure, progression-free survival, intermediatestage hepatocellular carcinoma, lenvatinib analyses. The median PFS time was 5.8, 3.2 and 2.4 months in the lenvatinib, sorafenib and TACE groups, respectively $(\mathrm{P}<0.001)$. In the Cox regression analysis, lenvatinib treatment and being within the up-to-seven criteria were identified as independent factors for PFS (lenvatinib, $\mathrm{P}<0.0001$; within up-to-seven, $\mathrm{P}=0.001)$. The decision-tree analysis revealed that patients beyond the up-to-seven criteria, treated with lenvatinib and with albumin-bilirubin (ALBI) grade 1 had a longer PFS time $(245.2 \pm 107.9$ days) than patients beyond the up-to-seven criteria, treated with lenvatinib and with ALBI grade 2 (147.1 \pm 78.6 days). Additionally, lenvatinib was independently associated with longer PFS time in patients with intermediate-stage HCC refractory to TACE. Therefore, lenvatinib may be recommended for patients who have intermediate-stage HCC refractory to TACE, ALBI grade 1 and who are within the up-to-seven criteria.

\section{Introduction}

Hepatocellular carcinoma (HCC) is the most common primary hepatic malignancy and a major cause of cancer-associated death worldwide in $2012(1,2)$. Although the prognosis of patients with early stage HCC has improved, the prognosis of patients with intermediate-stage HCC remains unsatisfactory (3). Transarterial chemoembolization (TACE) is the first-line treatment for patients with intermediate-stage HCC $(4,5)$. However, there is a high prevalence of HCC recurrence after TACE, resulting in a poor prognosis for patients with intermediate-stage $\mathrm{HCC}(3,6)$.

Repeated TACE has been a therapeutic strategy for HCC recurrence; however, this is associated with a decline in liver function and an ineffective response (7). The aforementioned condition is defined as 'refractory to TACE' or 'TACE failure' in the guidelines established by the Japan Society of 
Hepatology (JSH) (8) and the European Association for the Study of Liver (EASL) (3). The prognosis of patients with HCC refractory to TACE is poorer than that of patients with HCC non-refractory to TACE; therefore, other therapeutic strategies are being considered for these patients $(9,10)$.

Sorafenib, an oral multi-tyrosine kinase inhibitor (TKI), was the first alternative drug employed, and its efficacy and safety in patients with advanced HCC has been demonstrated in phase III study (11). Several studies reported that sorafenib improves overall survival (OS) time and time to progression in patients with intermediate- or advanced-stage HCC refractory to TACE $(9,10)$. Recently, an oral multi-kinase inhibitor, lenvatinib, was developed that targets vascular endothelial growth factor (VEGF) receptors 1-3 (VEGFR1-3) and fibroblast growth factor (FGF) receptors 1-4 (FGFR1-4) $(12,13)$. Although lenvatinib was non-inferior to sorafenib in terms of affecting the OS rate of patients with untreated advanced HCC, progression-free survival (PFS) was significantly longer in patients with $\mathrm{HCC}$ treated with lenvatinib than that in those treated with sorafenib (14). Therefore, lenvatinib has become the first-line systemic treatment for advanced HCC (14).

Recently, Ueshima et al (15) reported the beneficial effects of lenvatinib on the response rate in patients with early to advanced-stage HCC. Additionally, Kudo et al (16) reported the beneficial effects of lenvatinib on the OS rate in patients with intermediate-stage HCC with large or multinodular tumors exceeding the up-to-seven criteria (17). However, the beneficial effects of lenvatinib on OS time in patients with intermediate-stage HCC refractory to TACE remain unclear.

Since lenvatinib is a novel TKI with a limited observational period, the impact of lenvatinib on OS time cannot be evaluated. PFS time is defined as the time elapsed between treatment initiation and tumor progression or death from any cause, with censoring of patients lost to follow-up (18). An advantage of measuring PFS time is the ability to evaluate the effects of treatment even in a short observational period. Measuring PFS time is one of the ways to observe the efficacy of a novel treatment in clinical trials (19). Currently, PFS time is being reported as a surrogate of OS time in patients with HCC (20).

The aim of the present study was to investigate the difference in PFS time among patients with intermediate-stage HCC refractory to TACE treated with lenvatinib, sorafenib or TACE. Furthermore, the profiles associated with PFS time in patients with intermediate-stage HCC refractory to TACE were investigated using a data-mining analysis.

\section{Patients and methods}

Study design. The present retrospective study was performed in five institutions: Kurume University Hospital (Kurume, Japan), Yokokura Hospital (Miyama, Japan), Omuta City Hospital (Omuta, Japan), Yanagawa Hospital (Yanagawa, Japan) and Iwamoto Internal Medical Clinic (Kitakyushu, Japan). The current protocol conformed to the ethical guidelines of the 1975 Declaration of Helsinki, as reflected by the prior approval of the Ethical Committees of Kurume University School of Medicine. An opt-out approach was employed to obtain informed consent from patients, and personal information was protected during data collection.
Inclusion and exclusion criteria. The patient inclusion criteria for the present study were as follows: i) Diagnosis of intermediate-stage HCC refractory to TACE according to the Japan Society of Hepatology $(8,21)$; ii) age $>18$ years; iii) Eastern Cooperative Oncology Group performance status 0 (22); and iv) complete follow-up until death or study cessation (June 15, 2019). The patient exclusion criteria were as follows: i) A history of malignant tumors other than HCC in the 5 years preceding the present study; ii) participation in any clinical trial; iii) history of pretreatment with TKIs; iv) Child-Pugh class B or C (23); v) creatinine levels $>1.5 \mathrm{mg} / \mathrm{dl}$ (normal level $0.5-1.3 \mathrm{mg} / \mathrm{dl}$ ); vi) infiltrative $\mathrm{HCC}$, defined as a true infiltration of tumor cells into the liver parenchyma, a confluence of tiny nodules or both in computed tomography (CT) or magnetic resonance imaging (MRI) scans (24); vii) the presence of portal vein thrombosis or extrahepatic metastasis; viii) the presence of ascites; ix) esophageal varices with high risk of rupture; and $\mathrm{x})$ a history of choledochojejunostomy or liver transplantation.

Patients. A total of 641 consecutive patients with HCC who underwent TACE between Jan 1, 2009, and Feb 31, 2017, were registered; data cut-off for this analysis was June 15, 2019. Intermediate-stage HCC refractory to TACE was observed in 210 patients, and 39 patients with Child-Pugh class B or C were excluded from the analysis. Therefore, a total of 171 patients were enrolled in the present study. These patients were classified into three groups according to their treatment for HCC: The lenvatinib (Eisai Co., Ltd, Tokyo; n=45), sorafenib (Bayer Yakuhin, Ltd., Osaka, Japan; n=53) and TACE (n=73) groups (Fig. 1).

Diagnosis and staging of HCC. HCC was diagnosed using a combination of following serum markers and imaging: $\alpha$-fetoprotein (AFP) and des- $\gamma$-carboxy prothrombin (DCP), and imaging procedures, including ultrasonography, CT and MRI scans. HCC was classified using the Barcelona Clinic Liver Cancer (BCLC) staging system (4).

Definition of HCC refractory to TACE. HCC refractory to TACE was defined as previously described (8). Briefly, in the present study, HCC refractory to TACE was defined if any of following conditions were fulfilled: i) $\geq 2$ consecutive ineffective responses of treated tumors (viable lesions $>50 \%$ ); ii) $\geq 2$ consecutive progressive increases in total tumor count; or iii) continuous elevation in AFP or DCP levels after TACE.

Measurement of neutrophil to lymphocyte ratio (NLR). NLR was calculated by the ratio of neutrophil count to lymphocyte count: By dividing neutrophil count by lymphocyte which were the fraction of white blood cell (25).

Treatment for HCC refractory to TACE. The treatment strategy for HCC refractory to TACE was based on previous reports $(10,26)$. Sorafenib was administrated for patients with refractory to TACE; however, patients who refused sorafenib were treated with TACE. After lenvatinib was approved in Japan, 45 patients were treated with lenvatinib instead of sorafenib. After obtaining written informed consent from each patient, lenvatinib was orally administered at $12 \mathrm{mg} /$ day in patients with bodyweight $\geq 60 \mathrm{~kg}$ or $8 \mathrm{mg} /$ day in patients with bodyweight $<60 \mathrm{~kg}$. Sorafenib was orally administered at $400 \mathrm{mg}$ twice 


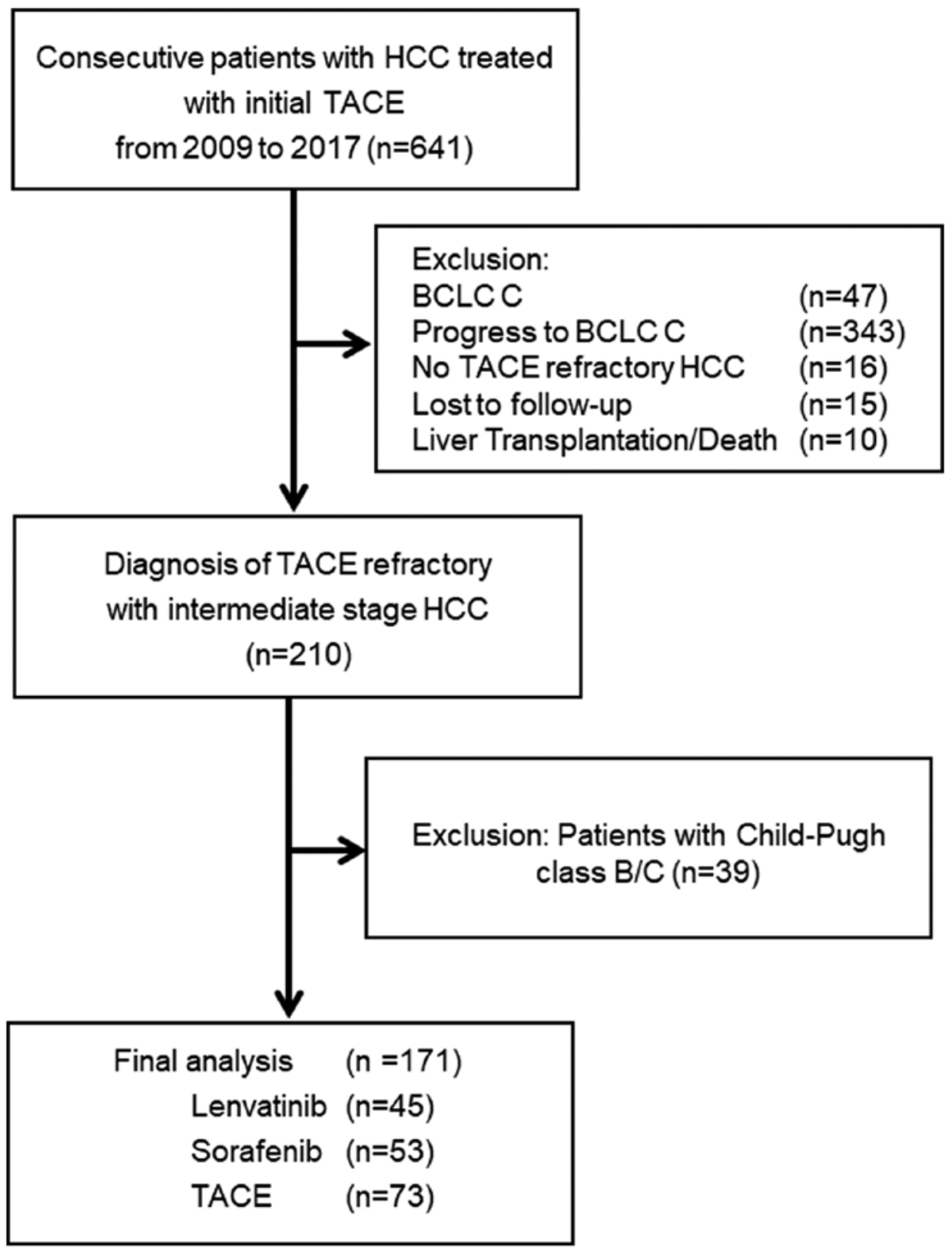

Figure 1. A total of 641 consecutive patients with HCC underwent TACE between Jan 1, 2009, and Feb 31, 2017. In the course of the study selection, 470 patients were excluded, and the remaining 171 patients with HCC were analyzed. Among the enrolled patients with HCC refractory to TACE, 45 patients were treated with lenvatinib, 53 patients with sorafenib and 73 patients with TACE. HCC, hepatocellular carcinoma; TACE, transarterial chemoembolization; BCLC, Barcelona Clinic Liver Cancer.

daily, regardless of bodyweight. Discontinuation and dose reduction of TKIs were based on the manufacturer's protocol (lenvatinib; Eisai Co., Ltd; sorafenib; Bayer Yakuhin, Ltd.).

TACE was performed by doctors with $>10$ years of experience in interventional therapy at the start of the present study (27). A catheter was inserted into the tumor-feeding artery; subsequently, anticancer drugs, such as epirubicin $(20-50 \mathrm{mg})$ or cisplatin $(20-50 \mathrm{mg})$ (depending on the size and number of tumors), were manually emulsified with lipiodol (Guerbet Japan K.K.) and administered, followed by embolization with 1-mm absorbable gelatin sponge particles (Nippon Kayaku Co., Ltd.).

Evaluation of the therapeutic response and the follow-up schedule. HCC was evaluated using CT or MRI scans 4-6 weeks after the initiation of treatment, and thereafter every 2-3 months until death or study cessation. The therapeutic response was evaluated using the Modified Response Evaluation Criteria in Solid Tumors (28): Complete response was defined as the disappearance of any intratumoral arterial enhancement in all target lesions; partial response (PR) was defined as a $\geq 30 \%$ decrease in the sum of the diameters of viable (contrast enhancement in the arterial phase) target lesions; progressive disease (PD) was defined as an increase of $\geq 20 \%$ in the sum of the diameters of viable (enhancing) target lesions, taking as reference the smallest sum of the diameters of viable (enhancing) target lesions recorded since the treatment started; and stable disease was defined as any other case that did not qualify for either PR or PD. Upon HCC recurrence, additional treatment was selected based on the evidence-based clinical practice guidelines of the BCLC staging system and treatment strategy (4). 
Table I. Characteristics of patients with HCC.

\begin{tabular}{|c|c|c|c|c|c|}
\hline Characteristic & $\begin{array}{l}\text { Total patients } \\
\quad(\mathrm{n}=171)\end{array}$ & $\begin{array}{l}\text { Lenvatinib } \\
\quad(n=45)\end{array}$ & $\begin{array}{l}\text { Sorafenib } \\
(\mathrm{n}=53)\end{array}$ & $\begin{array}{l}\text { TACE } \\
(\mathrm{n}=73)\end{array}$ & P-value \\
\hline Age, years, median (range) & $72(36-90)$ & $75(45-89)$ & $73(54-86)$ & $74(51-92)$ & 0.53 \\
\hline Sex, female/male & $32 / 139$ & $5 / 40$ & $7 / 46$ & $20 / 53$ & 0.04 \\
\hline BMI, $\mathrm{kg} / \mathrm{m}^{2}$, median (range) & $\begin{array}{c}22.7 \\
(14.9-34.1)\end{array}$ & $\begin{array}{c}22.8 \\
(15.4-31.4)\end{array}$ & $\begin{array}{c}23.2 \\
(17.7-34.1)\end{array}$ & $\begin{array}{c}22 \\
(14.9-30.1)\end{array}$ & 0.08 \\
\hline Cause of HCC, HCV/HBV/other & $114 / 20 / 37$ & $25 / 9 / 11$ & $34 / 7 / 12$ & $55 / 4 / 14$ & 0.07 \\
\hline ALBI score, median (range) & $\begin{array}{c}-2.25 \\
(-3.31--1.46)\end{array}$ & $\begin{array}{c}-2.48 \\
(-3.28--1.53)\end{array}$ & $\begin{array}{c}-2.30 \\
(-3.09--1.57)\end{array}$ & $\begin{array}{c}-2.11 \\
(-3.31--1.46)\end{array}$ & 0.11 \\
\hline ALBI grade, $1 / 2 / 3$ & $50 / 121 / 0$ & $18 / 27 / 0$ & $13 / 40 / 0$ & $19 / 54 / 0$ & 0.18 \\
\hline Up-to-seven, beyond/within & $144 / 27$ & $39 / 6$ & $45 / 8$ & $60 / 13$ & 0.79 \\
\hline 1 & 3 & 1 & 0 & 2 & \\
\hline 2 & 7 & 2 & 0 & 5 & \\
\hline 3 & 6 & 3 & 1 & 2 & \\
\hline 4 & 22 & 5 & 9 & 8 & \\
\hline$>5$ & 133 & 34 & 43 & 56 & 0.44 \\
\hline Tumor size, median (range), mm & $27(11-127)$ & $31(11-127)$ & $27(11-101)$ & $25(11-85)$ & 0.27 \\
\hline $\begin{array}{l}\text { Diabetes mellitus, } \\
\text { presence/absence }\end{array}$ & 72/99 & $21 / 24$ & $25 / 28$ & $26 / 47$ & 0.33 \\
\hline CRP, median (range), $\mathrm{mg} / \mathrm{dl}$ & $\begin{array}{c}0.11 \\
(0-5.67)\end{array}$ & $\begin{array}{c}0.18 \\
(0.01-2.46)\end{array}$ & $\begin{array}{c}0.1 \\
(0.04-4.25)\end{array}$ & $\begin{array}{c}0.1 \\
(0-5.67)\end{array}$ & 0.23 \\
\hline NLR, median (range) & $\begin{array}{c}2.25 \\
(0.1-29.1)\end{array}$ & $\begin{array}{c}2.16 \\
(0.1-9.1)\end{array}$ & $\begin{array}{c}2.23 \\
(0.5-29.1)\end{array}$ & $\begin{array}{c}2.36 \\
(0.48-11.9)\end{array}$ & 0.23 \\
\hline AFP, median (range), $\mathrm{ng} / \mathrm{ml}$ & $\begin{array}{c}54.4 \\
(1.8-113534)\end{array}$ & $\begin{array}{c}34.1 \\
(1.9-2675)\end{array}$ & $\begin{array}{c}50.8 \\
(2.8-30772)\end{array}$ & $\begin{array}{c}64.5 \\
(1.8-113534)\end{array}$ & 0.35 \\
\hline $\begin{array}{l}\text { DCP, median (range), } \\
\mathrm{mAU} / \mathrm{ml}\end{array}$ & $\begin{array}{c}218 \\
(7.1-45929)\end{array}$ & $\begin{array}{c}137 \\
(12-20657)\end{array}$ & $\begin{array}{c}406 \\
(19-23197)\end{array}$ & $\begin{array}{c}395 \\
(7.1-45929)\end{array}$ & 0.14 \\
\hline $\begin{array}{l}\text { Creatinine, median (range), } \\
\mathrm{mg} / \mathrm{dl}\end{array}$ & $\begin{array}{c}0.81 \\
(0.33-1.49)\end{array}$ & $\begin{array}{c}0.83 \\
(0.54-1.49)\end{array}$ & $\begin{array}{c}0.82 \\
(0.41-1.48)\end{array}$ & $\begin{array}{c}0.76 \\
(0.33-1.4)\end{array}$ & 0.42 \\
\hline $\begin{array}{l}\text { eGFR, median (range), } \\
\mathrm{ml} / \mathrm{min} / 1.73 \mathrm{~m}^{2}\end{array}$ & $\begin{array}{c}70.6 \\
(31.7-150)\end{array}$ & $\begin{array}{c}68.6 \\
(31.7-118)\end{array}$ & $\begin{array}{c}70.6 \\
(31.8-150)\end{array}$ & $\begin{array}{c}74.7 \\
(38.5-143)\end{array}$ & 0.73 \\
\hline
\end{tabular}

Note. Data are expressed as median (range), or number. TACE, transarterial chemoembolization; BMI, body mass index; HCC, hepatocellular carcinoma; HBV, hepatitis B virus; HCV, hepatitis C virus; ALBI, albumin-bilirubin; CRP, C reactive protein; NLR, neutrophil to lymphocyte ratio; AFP, $\alpha$-fetoprotein; DCP, des- $\gamma$-carboxy prothrombin; eGFR, estimate glomerular filtration rate.

Table II. Treatment response rate for hepatocellular carcinoma refractory to TACE in the lenvatinib $(n=43)$, sorafenib $(n=53)$ and TACE $(n=73)$ groups.

\begin{tabular}{lccc}
\hline $\begin{array}{l}\text { Response } \\
\text { category }\end{array}$ & $\begin{array}{c}\text { Lenvatinib, } \\
\mathrm{n}(\%)\end{array}$ & $\begin{array}{c}\text { Sorafenib, } \\
\mathrm{n}(\%)\end{array}$ & $\begin{array}{c}\text { TACE, } \\
\mathrm{n}(\%)\end{array}$ \\
\hline Complete response & $1(2)$ & $0(0)$ & $1(1)$ \\
Partial response & $18(40)$ & $1(2)$ & $4(6)$ \\
Stable disease & $21(47)$ & $30(56)$ & $9(12)$ \\
Progressive disease & $5(11)$ & $22(42)$ & $59(81)$ \\
Objective response rate & $19(42)$ & $1(2)$ & $5(7)$ \\
Disease control rate & $40(89)$ & $31(58)$ & $14(19)$
\end{tabular}

TACE, transarterial chemoembolization.
Safety evaluation. Adverse events (AEs) and serious adverse events (SAEs) were monitored and recorded. AEs were assessed according to the National Cancer Institute Common Terminology Criteria for Adverse Events (CTCAE) v4.0 (29). In the lenvatinib and sorafenib groups, when a patient developed any grade $\geq 3 \mathrm{SAE}$ (according to CTCAE), or if any unacceptable grade 2 drug-related AE occurred, the drug dose was reduced or discontinued. Additionally, the effects of the treatments on liver function were evaluated by measuring changes in the albumin-bilirubin (ALBI) score. ALBI score was calculated as previously described (30) based on serum albumin and total bilirubin levels; ALBI-score $=\left[\log _{10}\right.$ bilirubin $(\mu \mathrm{mol} / \mathrm{l}) \mathrm{x} 0.66]+[$ albumin $(\mathrm{g} / \mathrm{l}) \mathrm{x}-0.085]$, and was graded as following: $\leq-2.60=$ ALBI grade $1,>-2.60$ to $\leq-1.39=$ ALBI grade 2, $>-1.39=$ ALBI grade 3 ). 
Table III. Univariate and multivariate analyses of factors potentially associated with progression-free survival.

\begin{tabular}{|c|c|c|c|c|}
\hline \multirow[b]{2}{*}{ Variable } & \multirow{2}{*}{$\begin{array}{c}\text { Univariate analysis } \\
\text { P-value }\end{array}$} & \multicolumn{3}{|c|}{ Multivariate analysis } \\
\hline & & Odds ratio & $95 \% \mathrm{CI}$ & P-value \\
\hline Age & 0.150 & & & \\
\hline Sex, female vs. male & 0.747 & & & \\
\hline $\mathrm{BMI},<22 \mathrm{vs} . \geq 22 \mathrm{~kg} / \mathrm{m}^{2}$ & 0.255 & & & \\
\hline Cause of HCC, HCV vs. HBV vs. other & 0.117 & & & \\
\hline ALBI grade, 1 vs. 2 & 0.028 & 0.81 & $0.559-1.145$ & 0.239 \\
\hline Up-to-seven criteria, within vs. beyond & 0.029 & 0.49 & $0.31-0.77$ & 0.001 \\
\hline Diabetes mellitus, (presence vs. absence & 0.164 & & & \\
\hline NLR,$<2.25$ vs. $\geq 2.25$ & 0.114 & & & \\
\hline \multicolumn{5}{|l|}{ Treatment } \\
\hline Lenvatinib & $<0.001$ & 0.36 & $0.237-0.533$ & $<0.001$ \\
\hline Sorafenib & 0.439 & & & \\
\hline AFP, $<200 \mathrm{vs} . \geq 200 \mathrm{ng} / \mathrm{ml}$ & 0.119 & & & \\
\hline DCP,$<200$ vs. $\geq 200 \mathrm{mAU} / \mathrm{ml}$ & 0.117 & & & \\
\hline
\end{tabular}

BMI, Body Mass Index; HCC, hepatocellular carcinoma; HBV, hepatitis B virus; HCV, hepatitis C virus; ALBI, albumin-bilirubin; NLR, neutrophil to lymphocyte ratio; TACE, transarterial chemoembolization; AFP, $\alpha$-fetoprotein; DCP, des- $\gamma$-carboxy prothrombin.

Statistical analysis. All data are presented as the number or median (range). All statistical analyses were performed using JMP Pro v13 (SAS Institute, Inc.). Categorical variables were compared using the $\chi^{2}$ test, and continuous variables were compared using one-way ANOVA with Scheffe's post hoc test. OS and PFS rates were compared between the groups using the log-rank test or Bonferroni method. Variable factors associated with PFS were analyzed using decision-tree analyses. Univariate and multivariate analyses were conducted using the Cox proportional hazards model to identify risk factors associated with PFS. $\mathrm{P}<0.05$ (two-tailed) was considered to indicate a statistically significant difference.

\section{Results}

Patient characteristics. The characteristics of the enrolled patients are shown in Table I. The median age was 72 years, and $18.1 \%(31 / 171)$ of the patients were female. The median BMI was $22.7 \mathrm{~kg} / \mathrm{m}^{2}$. ALBI grade 1 was observed in $29.2 \%$ $(50 / 171)$ of patients, and the median NLR was 2.25 . The median tumor size was $27 \mathrm{~mm}$, and 84.2\% (144/171) of patients were beyond the up-to-seven criteria, beyond 7 being the sum of the maximum size and number of tumors for any given HCC case (17) (Table I). In the TACE group, the female-to-male ratio was significantly higher than that in the lenvatinib and sorafenib groups (Table I). No significant differences were seen for age, BMI, etiology of liver disease, AFP level or NLR among the three groups. Furthermore, no significant differences were observed in the prevalence of beyond the up-to-seven criteria or ALBI grade among the three groups.

Treatment response for TACE refractory HCC. The overall objective response rate (ORR) was 42.2\% (19/45), 1.9\% (1/53) and $6.8 \%(5 / 73)$ in the lenvatinib, sorafenib and TACE groups, respectively, 1 month after treatment (Table II). The disease control rate (DCR) was $88.9 \%$ (40/45), 56.6\% (31/53) and $19 \%$ $(14 / 73)$ in the lenvatinib, sorafenib and TACE groups, respectively, 1 month after treatment (Table II).

Relative dose intensity (RDI) of lenvatinib for ORR. In the present study, the RDI was 80.7 and $73.3 \%$ at 4 and 8 weeks, respectively. There was a significant difference in ORR between patients in the 8 week-RDI $\geq 75 \%$ group and the 8 week-RDI $<75 \%$ group [ORR, 10/24 (41.7\%) vs. 5/21 (23.8\%), respectively; $\mathrm{P}=0.02$ ] (date not shown).

PFS time in the lenvatinib, sorafenib and TACE groups. The median PFS time was 5.8, 3.2 and 2.8 months in the lenvatinib, sorafenib and TACE groups, respectively (Fig. 2). Among the three groups, the longest median PFS time was observed in the lenvatinib group. The median PFS time in the lenvatinib group was significantly longer than that in the sorafenib and TACE groups [lenvatinib vs. sorafenib: Hazard ratio (HR), 0.56; 95\% confidence interval (CI), 0.36-0.88; $\mathrm{P}=0.01$; lenvatinib vs. TACE: HR, 0.23; 95\% CI, 0.15-0.36; $\mathrm{P}<0.001]$.

Univariate and multivariate analyses of factors associated with PFS. Treatment with lenvatinib, being within the up-to-seven criteria and ALBI grade 1 were selected as variables via univariate analysis. In the multivariate analysis, treatment with lenvatinib and being within up-to-seven were identified as independent factors for PFS (Table III).

Decision-tree analysis for PFS. The period of PFS was $130 \pm 114.5$ days at the study censor time. To determine the profile for PFS, a decision-tree analysis was performed. 


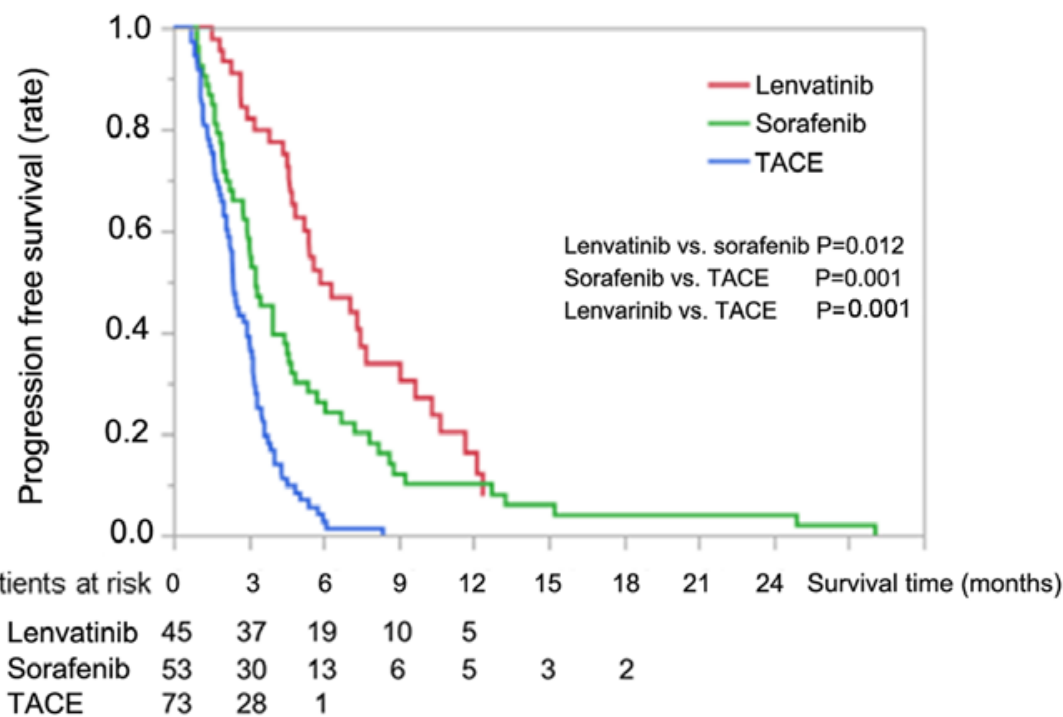

Figure 2. Kaplan-Meier curves for progression-free survival in the lenvatinib, sorafenib and TACE groups. TACE, transarterial chemoembolization.

\section{Model building

$$
\mathrm{n}=171
$$

\section{【Period of PFS $130 \pm 114.5$ days】}

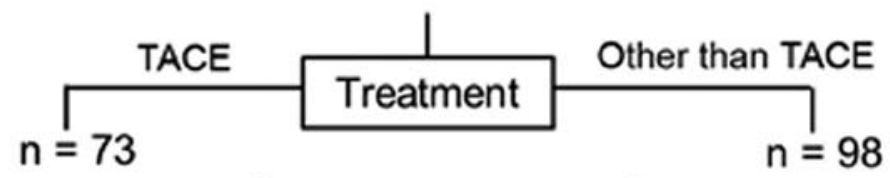

【80.3 \pm 44.7 days】

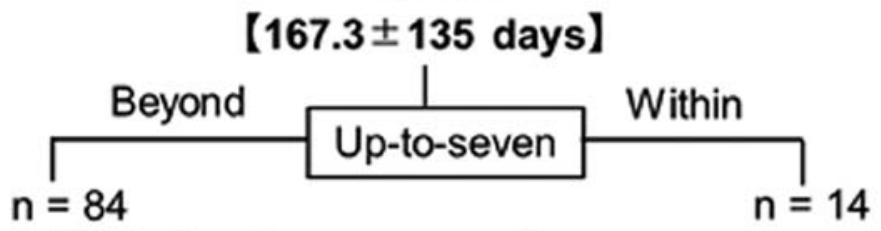

【148.8 \pm 104.1 days】

【278.6 \pm 226.3 days】

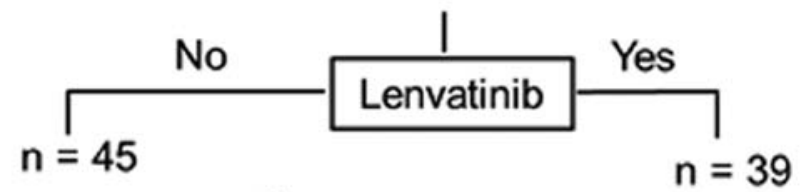

【117.6 \pm 96.8 days】

【184.8 \pm 101.8 days】

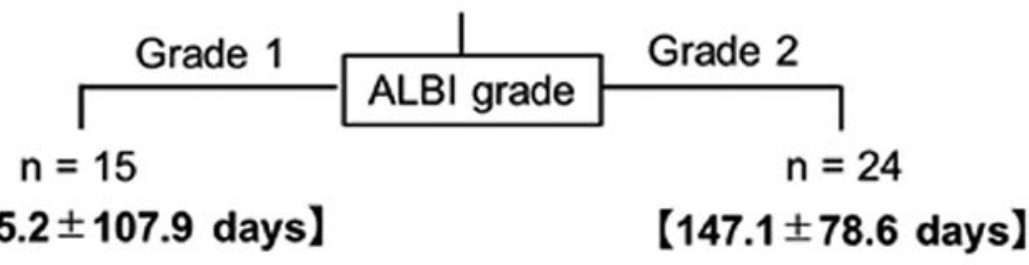

Figure 3. Decision-tree algorithm for the periods of PFS. The period of PFS was expressed as the mean \pm SD. TACE, transarterial chemoembolization; ALBI, albumin-bilirubin; PFS, progression-free survival.

Treatment for HCC was selected as the variable for the initial split. PFS time was $80.3 \pm 44.7$ days in patients treated with TACE (Fig. 3); in patients receiving treatments other than TACE and beyond the up-to-seven criteria, the PFS time was $148.8 \pm 104.1$ days. In patients beyond the up-to-seven criteria, lenvatinib treatment was selected as the third split. The PFS time was $184.8 \pm 101.8$ and $117.6 \pm 96.8$ days in patients with and without lenvatinib treatment, respectively (Fig. 3). In addition, the ALBI grade was selected as the fourth split in patients treated with lenvatinib. The PFS time was $245.2 \pm 107.9$ and 147.1 \pm 78.6 days in patients with ALBI grades 1 and 2, respectively. 
Table IV. Adverse events associated with lenvatinib $(n=45)$, sorafenib $(n=53)$ and TACE $(n=73)$ treatment (grade $\geq 3)$.

\begin{tabular}{lrrr}
\hline Adverse event & $\begin{array}{c}\text { Lenvatinib, } \\
\%(\mathrm{n})\end{array}$ & $\begin{array}{c}\text { Sorafenib, } \\
\%(\mathrm{n})\end{array}$ & $\begin{array}{c}\text { TACE, } \\
\%(\mathrm{n})\end{array}$ \\
\hline Fatigue & $13.3(6)$ & $7.5(4)$ & $0.0(0)$ \\
Hypertension & $15.5(7)$ & $3.7(2)$ & $0.0(0)$ \\
HFSR & $4.4(2)$ & $16.9(9)$ & $0.0(0)$ \\
Loss of appetite & $2.2(1)$ & $1.9(1)$ & $0.0(0)$ \\
Diarrhea & $8.8(4)$ & $7.5(4)$ & $0.0(0)$ \\
Urine protein & $11.1(5)$ & $0.0(0)$ & $0.0(0)$ \\
Ascites & $0.0(0)$ & $3.7(2)$ & $9.6(7)$ \\
Elevated aspartate & & & \\
aminotransferase level & $0.0(0)$ & $2.0(1)$ & $8.0(6)$ \\
Digestive track bleeding & $2.2(1)$ & $3.7(2)$ & $0.0(0)$ \\
\hline
\end{tabular}

TACE, transarterial chemoembolization; HFSR, hand-foot-skin-reaction.

OS in the lenvatinib, sorafenib and TACE groups. In the Lenvatinib group, an estimated median OS was not observed within the present study (Fig. S1).

Treatment response and medication periods with lenvatinib based on ALBI grade. The ORRs were $66.7 \%(12 / 18)$ and $25.9 \%(7 / 27)$ in patients with ALBI grade 1 and 2 , respectively (data not shown). The median medication period with lenvatinib in patients with ALBI grades 1 and 2 was 11.2 and 5.9 months, respectively ( $\mathrm{P}=0.002$; Fig. S2).

Additional treatments in each group. In the TACE group, 44 patients were treated with repeated TACE, 10 patients were treated with repeated TACE followed by sorafenib after reaching BCLC stage $\mathrm{C}, 9$ patients were treated with hepatic arterial infusion chemotherapy (HAIC) and 10 patients received palliative care after obtaining informed consent, as hepatic function deteriorated to Child-Pugh class C. In the sorafenib group, 29 patients were treated with TACE, 13 patients with HAIC, 3 patients with regorafenib and 8 patients received palliative care. In the lenvatinib group, 16 patients were treated with TACE, 8 patients were treated with HAIC, 7 patients were continuously treated with lenvatinib even after disease progression, 3 patients were treated with sorafenib, 1 patient was treated with radiation therapy and 8 patients were treated with lenvatinib alone. Furthermore, in patients whose hepatic function deteriorated to Child-Pugh class B, TACE was performed after obtaining informed consent; in patients whose hepatic function deteriorated to Child-Pugh class $\mathrm{C}$, palliative care was selected after obtaining informed consent.

AEs from the treatment (grade $\geq 3$ ). AEs (grade $\geq 3$ ) were determined by the attending physician for the lenvatinib, sorafenib and TACE groups, and are shown in Table IV. In the lenvatinib group, the most common AEs were hypertension and fatigue, which occurred in $15.6 \%$ (7/45) and $13.3 \%$ (6/45) of the patients, respectively. In the sorafenib group, hand-foot-skin-reactions occurred in $17.0 \%(9 / 53)$ of the patients. In the TACE group, ascites occurred in $9.6 \%(7 / 73)$ of the patients.

\section{Discussion}

In the present study, both lenvatinib treatment and the up-to-seven criteria were independently associated with longer PFS time in patients with intermediate-stage HCC refractory to TACE. Furthermore, it was revealed that lenvatinib may be recommended for patients who have intermediate-stage HCC refractory to TACE, ALBI grade 1 and who are within the up-to-seven criteria.

The present study demonstrated an ORR, DCR and median PFS time of $42.2 \%, 88.9 \%$ and 5.8 months, respectively, in patients with HCC refractory to TACE treated with lenvatinib. A previous study reported a median PFS time of $\sim 3.5$ months in patients with unresectable HCC refractory to TACE (31). The median PFS time of patients treated with lenvatinib in the present study was longer than that of patients with unresectable HCC treated with TACE alone. Currently, HCC refractory to TACE or with TACE failure is not considered appropriate for repeated TACE $(9,10)$. The ORR, the DCR and the median PFS time were reported to be $40.6 \%, 73.8 \%$ and 7.4 months, respectively, in patients with unresectable HCC treated with lenvatinib $(14,32)$; however, the impact of lenvatinib on PFS time remains unclear in patients with HCC refractory to TACE. Therefore, the efficacy of lenvatinib on PFS time in patients with intermediate-stage $\mathrm{HCC}$ refractory to TACE was analyzed. Although OS could not be assessed in the present study as the estimated median survival time could not be reached, PFS time was used as a surrogate marker for OS (20). Accordingly, lenvatinib may prolong survival time in patients with intermediate-stage HCC refractory to TACE.

Although the beneficial effects of lenvatinib have been previously reported in patients with advanced-stage HCC (14), in the present study, lenvatinib was independently associated with longer PFS time in patients with intermediate-stage HCC refractory to TACE. Additionally, the median PFS time in the lenvatinib group was significantly longer than that in the sorafenib and TACE groups. In HCC refractory to TACE, a potential pathogenic mechanism is presumed to be a neoangiogenic reaction (33). Serum VEGF and FGF levels are reportedly increased following treatment with TACE and seem to affect patient survival (33). Lenvatinib is a potent inhibitor of VEGFR1-3 and pro-oncogenic receptor tyrosine kinases, including FGFR1-4 $(12,34,35)$. In addition, the $\mathrm{IC}_{50}$ of lenvatinib for VEGFR and FGFR is lower than that of sorafenib (36). Additionally, lenvatinib is more cost-effective compared with sorafenib for the first-line treatment of unresectable HCC (37). Therefore, lenvatinib may be more beneficial than sorafenib in patients with HCC refractory to TACE. However, to the best of our knowledge, there are no available biomarkers to help inform the choice of first-line treatment, and the optimum choice of treatment in clinical practice remains unclear.

The present study demonstrated that patients with ALBI grade 1 treated with lenvatinib exhibited improved PFS times compared with those with ALBI grade 2. Although there are no studies on PFS time in patients with TACE-refractory HCC treated with lenvatinib, Hiraoka et al (32) reported that good liver function indicated by ALBI grade 1 is the best indication for lenvatinib. Additionally, Ueshima et al (15) reported that ALBI grade 1 predicts a higher response rate than ALBI grade 2 . Serum albumin is known as a pharmacokinetic factor 
of lenvatinib (38); therefore, patients with ALBI grade 1 may exhibit improved drug sensitivity to lenvatinib compared with patients with ALBI grade 2. In addition, lenvatinib compliance is lower in patients with ALBI grade 2 than that in patients with ALBI grade $1(15,32)$. In the present study, the ORR was higher in patients with ALBI grade 1 than that in patients with ALBI grade 2. Furthermore, the medication period of lenvatinib in the ALBI grade 1 group was significantly longer than that in the ALBI grade 2 group. Therefore, the higher ORR and longer medication period of lenvatinib may contribute to longer PFS times in patients with ALBI grade 1 intermediate-stage HCC refractory to TACE.

The present study had several limitations. First, the study design was retrospective, and the sample size was small. Second, there was selection bias for the classification of the lenvatinib, sorafenib and TACE groups. Third, the TACE techniques and devices used in the present study are not comparable with the latest TACE techniques, such as balloon-TACE (39), drug-eluting-beads-TACE (40) and next-generation micro-catheters. Fourth, other tumor criteria were not evaluated, including the up-to-eleven criteria (41). Fifth, the impact on OS was not evaluated due to the limited observational period with lenvatinib treatment. Since OS is affected by further therapies after first-line treatment, sequential therapies should be considered following ineffective treatment for HCC $(42,43)$. Further randomized, controlled and prospective studies are required to establish the indications for lenvatinib treatment in patients with intermediate-stage HCC refractory to TACE.

In conclusion, lenvatinib treatment was independently associated with longer PFS time in patients with intermediate-stage HCC refractory to TACE. Furthermore, the present study revealed that lenvatinib may be recommended for patients who have intermediate-stage HCC refractory to TACE, ALBI grade 1 and who are within the up-to-seven criteria.

\section{Acknowledgements}

The authors would like to thank Dr Ryoko Kuromatsu (Division of Gastroenterology, Department of Medicine, Kurume University School of Medicine), Dr Shusuke Okamura (Division of Gastroenterology, Department of Medicine, Kurume University School of Medicine), Dr Yu Noda (Division of Gastroenterology, Department of Medicine, Kurume University School of Medicine) and Dr Naoki Kamachi (Division of Gastroenterology, Department of Medicine, Kurume University School of Medicine) for the clinical management of patients. A part of this abstract was presented at the 70th Annual Meeting of the American Association for Liver Diseases; abstract no. 367, Nov 8-12, 2019 in Boston, MA).

\section{Funding}

No funding was received.

\section{Availability of data and materials}

The datasets used and/or analyzed during the present study are available from the corresponding author on reasonable request.

\section{Authors' contributions}

SS and TK participated in the study conception and design, in the acquisition and interpretation of data, and in the drafting of the manuscript. MT, HI, KM, EM, HSuz, TN, TS, MN, HSug, TY, YY and KN participated in the acquisition of data. MT participated in analysis, interpretation of data and drafting of manuscript. HK and TT participated in the study conception, design and critical revision.

\section{Ethics approval and consent to participate}

The present study was approved by the Ethical Committee of Kurume University School of Medicine (approval no. 18298), and an opt-out approach was used to obtain informed consent from the patients.

\section{Patient consent for publication}

Not applicable.

\section{Competing interests}

Takumi Kawaguchi received an honorarium (lecture fee) from Mitsubishi Tanabe Pharma Corporation and Otsuka Pharmaceutical Co., Ltd.

\section{References}

1. Llovet JM, Montal R, Sia D and Finn RS: Molecular therapies and precision medicine for hepatocellular carcinoma. Nat Rev Clin Oncol 15: 599-616, 2018.

2. Torre LA, Bray F, Siegel RL, Ferlay J, Lortet-Tieulent J and Jemal A: Global cancer statistics, 2012. CA Cancer J Clin 65: 87-108, 2015.

3. European Association for the Study of the Liver.Electronic address: easloffice@easloffice.eu; European Association for the Study of the Liver: EASL Clinical Practice Guidelines: Management of hepatocellular carcinoma. J Hepatol 69: 182-236, 2018.

4. Forner A, Reig M and Bruix J: Hepatocellular carcinoma. Lancet 391: 1301-1314, 2018.

5. Bruix J, Sherman M and American Association for the Study of Liver Diseases: Management of hepatocellular carcinoma: An update. Hepatology 53: 1020-1022, 2011.

6. Heimbach JK, Kulik LM, Finn RS, Sirlin CB, Abecassis MM, Roberts LR, Zhu AX, Murad MH and Marrero JA: AASLD guidelines for the treatment of hepatocellular carcinoma. Hepatology 67: 358-380, 2018.

7. Hiraoka A, Kumada T, Kudo M, Hirooka M, Koizumi Y, Hiasa Y, Tajiri K, Toyoda H, Tada T, Ochi H, et al: Hepatic function during repeated TACE procedures and prognosis after introducing sorafenib in patients with unresectable hepatocellular carcinoma: Multicenter Analysis. Dig Dis 35: 602-610, 2017.

8. Kudo M, Matsui O, Izumi N, Kadoya M, Okusaka T, Miyayama S, YamakadoK,Tsuchiya K,Ueshima K,Hiraoka A, et al: Transarterial chemoembolization failure/refractoriness: JSH-LCSGJ criteria 2014 update. Oncology 87 (Suppl 1): 22-31, 2014

9. Ogasawara S, Chiba T, Ooka Y, Kanogawa N, Motoyama T, Suzuki E, Tawada A, Kanai F, Yoshikawa M and Yokosuka O: Efficacy of sorafenib in intermediate-stage hepatocellular carcinoma patients refractory to transarterial chemoembolization. Oncology 87: 330-341, 2014.

10. Ikeda M, Mitsunaga S, Shimizu S, Ohno I, Takahashi H, Okuyama H, Kuwahara A, Kondo S, Morizane C, Ueno H, et al: Efficacy of sorafenib in patients with hepatocellular carcinoma refractory to transcatheter arterial chemoembolization. J Gastroenterol 49: 932-940, 2014.

11. Llovet JM, Ricci S, Mazzaferro V, Hilgard P, Gane E, Blanc JF, de Oliveira AC, Santoro A, Raoul JL, Forner A, et al: Sorafenib in advanced hepatocellular carcinoma. N Engl J Med 359: 378-390, 2008 . 
12. Tohyama O, Matsui J, Kodama K, Hata-Sugi N, Kimura T, Okamoto K, Minoshima Y, Iwata M and Funahashi Y: Antitumor activity of lenvatinib (e7080): An angiogenesis inhibitor that targets multiple receptor tyrosine kinases in preclinical human thyroid cancer models. J Thyroid Res 2014: 638747, 2014.

13. Yamamoto Y, Matsui J, Matsushima T, Obaishi H, Miyazaki K, Nakamura K, Tohyama O, Semba T, Yamaguchi A, Hoshi SS, et al: Lenvatinib, an angiogenesis inhibitor targeting VEGFR/FGFR, shows broad antitumor activity in human tumor xenograft models associated with microvessel density and pericyte coverage. Vasc Cell 6: 18, 2014.

14. Kudo M, Finn RS, Qin S, Han KH, Ikeda K, Piscaglia F, Baron A, Park JW, Han G, Jassem J, et al: Lenvatinib versus sorafenib in first-line treatment of patients with unresectable hepatocellular carcinoma: A randomised phase 3 non-inferiority trial. Lancet 391: 1163-1173, 2018.

15. Ueshima K, Nishida N, Hagiwara $S$, Aoki $T$, Minami $T$, Chishina H, Takita M, Minami Y, Ida H, Takenaka M, et al: Impact of baseline ALBI grade on the outcomes of hepatocellular carcinoma patients treated with lenvatinib: A multicenter study. Cancers (Basel) 11: 952, 2019.

16. Kudo M, Ueshima K, Chan S, Minami T, Chishina H, Aoki T, Takita M, Hagiwara S, Minami Y, Ida H, et al: Lenvatinib as an initial treatment in patients with intermediate-stage hepatocellular carcinoma beyond up-to-seven criteria and child-pugh a liver function: A proof-of-concept study. Cancers (Basel) 11: 1084, 2019.

17. D'Amico F, Schwartz M, Vitale A, Tabrizian P, Roayaie S Thung S, Guido M, del Rio Martin J, Schiano T and Cillo U: Predicting recurrence after liver transplantation in patients with hepatocellular carcinoma exceeding the up-to-seven criteria. Liver Transpl 15: 1278-1287, 2009.

18. Saad ED and Katz A: Progression-free survival and time to progression as primary end points in advanced breast cancer: Often used, sometimes loosely defined. Ann Oncol 20: 460-464, 2009.

19. Forsythe A, Chandiwana D, Barth J, Thabane M, Baeck J, Shor A and Tremblay G: Is progression-free survival a more relevant endpoint than overall survival in first-line HR+/HER2-metastatic breast cancer? Cancer Manag Res 10: 1015-1025, 2018.

20. Llovet JM, Montal R and Villanueva A: Randomized trials and endpoints in advanced HCC: Role of PFS as a surrogate of survival. J Hepatol 70: 1262-1277, 2019.

21. Kokudo N, Hasegawa K, Akahane M, Igaki H, Izumi N, Ichida T, Uemoto S, Kaneko S, Kawasaki S, Ku Y, et al: Evidence-based Clinical Practice Guidelines for Hepatocellular Carcinoma: The Japan Society of Hepatology 2013 update (3rd JSH-HCC Guidelines). Hepatol Res 45: 2015.

22. Oken MM, Creech RH, Tormey DC, Horton J, Davis TE, McFadden ET and Carbone PP: Toxicity and response criteria of the eastern cooperative oncology group. Am J Clin Oncol 5: 649-655, 1982

23. Cholongitas E, Papatheodoridis GV, Vangeli M, Terreni N, Patch D and Burroughs AK: Systematic review: The model for end-stage liver disease-should it replace Child-Pugh's classification for assessing prognosis in cirrhosis? Aliment Pharmacol Ther 22: 1079-1089, 2005.

24. Chernyak V, Fowler KJ, Kamaya A, Kielar AZ, Elsayes KM, Bashir MR, Kono Y, Do RK, Mitchell DG, Singal AG, et al: Liver imaging reporting and data system (LI-RADS) Version 2018: Imaging of hepatocellular carcinoma in at-risk patients. Radiology 289: 816-830, 2018

25. Tada T, Kumada T, Hiraoka A, Michitaka K, Atsukawa M, Hirooka M, Tsuji K, Ishikawa T, Takaguchi K, Kariyama K, et al: Neutrophil-to-lymphocyte ratio is associated with survival in patients with unresectable hepatocellular carcinoma treated with lenvatinib. Liver Int 40: 968-976, 2020

26. Arizumi T, Ueshima $\mathrm{K}$, Minami T, Kono $\mathrm{M}$, Chishina $\mathrm{H}$, Takita M, Kitai S, Inoue T, Yada N, Hagiwara S, et al: Effectiveness of sorafenib in patients with transcatheter arterial chemoembolization (TACE) refractory and intermediate-stage hepatocellular carcinoma. Liver Cancer 4: 253-262, 2015.

27. Shimose S, Tanaka M, Iwamoto H, Niizeki T, Shirono T, Aino H, Noda Y, Kamachi N, Okamura S, Nakano M, et al: Prognostic impact of transcatheter arterial chemoembolization (TACE) combined with radiofrequency ablation in patients with unresectable hepatocellular carcinoma: Comparison with TACE alone using decision-tree analysis after propensity score matching. Hepatol Res 49: 919-928, 2019.

28. Lencioni R and Llovet JM: Modified RECIST (mRECIST) assessment for hepatocellular carcinoma. Semin Liver Dis 30: 52-60, 2010
29. Atkinson TM, Ryan SJ, Bennett AV, Stover AM, Saracino RM, Rogak LJ, Jewell ST, Matsoukas K, Li Y and Basch E: The association between clinician-based common terminology criteria for adverse events (CTCAE) and patient-reported outcomes (PRO): A systematic review. Support Care Cancer 24: 3669-3676, 2016.

30. Johnson PJ, Berhane S, Kagebayashi C, Satomura S, Teng M, Reeves HL, O'Beirne J, Fox R, Skowronska A, Palmer D, et al: Assessment of liver function in patients with hepatocellular carcinoma: A new evidence-based approach-the ALBI grade. J Clin Oncol 33: 550-558, 2015.

31. Ohki T, Sato K, Yamagami M, Ito D, Yamada T, Kawanishi K, Kojima K, Seki M, Toda N and Tagawa K: Efficacy of transcatheter arterial chemoembolization followed by sorafenib for intermediate/advanced hepatocellular carcinoma in patients in Japan: A retrospective analysis. Clin Drug Investig 35: 751-759, 2015.

32. Hiraoka A, Kumada T, Atsukawa M, Hirooka M, Tsuji K, Ishikawa T, Takaguchi K, Kariyama K, Itobayashi E, Tajiri K, et al: Prognostic factor of lenvatinib for unresectable hepatocellular carcinoma in real-world conditions-Multicenter analysis. Cancer Med 8: 3719-3728, 2019.

33. Sergio A, Cristofori C, Cardin R, Pivetta G, Ragazzi R, Baldan A, Girardi L, Cillo U, Burra P, Giacomin A and Farinati F: Transcatheter arterial chemoembolization (TACE) in hepatocellular carcinoma (HCC): The role of angiogenesis and invasiveness. Am J Gastroenterol 103: 914-921, 2008.

34. Hoshi T, Watanabe Miyano S, Watanabe H, Sonobe RMK, Seki Y, Ohta E, Nomoto K, Matsui J and Funahashi Y: Lenvatinib induces death of human hepatocellular carcinoma cells harboring an activated FGF signaling pathway through inhibition of FGFR-MAPK cascades. Biochem Biophys Res Commun 513: 1-7, 2019.

35. Kimura T, Kato Y, Ozawa Y, Kodama K, Ito J, Ichikawa K, Yamada K, Hori Y, Tabata K, Takase K, et al: Immunomodulatory activity of lenvatinib contributes to antitumor activity in the Hepa1-6 hepatocellular carcinoma model. Cancer Sci 109: 3993-4002, 2018.

36. Adachi Y, Matsuki M, Watanabe H, Takase K, Kodama K, Matsui J, Funahashi Y and Nomoto K: Antitumor and antiangiogenic activities of lenvatinib in mouse xenograft models of vascular endothelial growth factor-induced hypervascular human hepatocellular carcinoma. Cancer Invest 37: 185-198, 2019.

37. Kobayashi M, Kudo M, Izumi N, Kaneko S, Azuma M, Copher R, Meier G, Pan J, Ishii M and Ikeda S: Cost-effectiveness analysis of lenvatinib treatment for patients with unresectable hepatocellular carcinoma (uHCC) compared with sorafenib in Japan. J Gastroenterol 54: 558-570, 2019.

38. Gupta A, Jarzab B, Capdevila J, Shumaker R and Hussein Z Population pharmacokinetic analysis of lenvatinib in healthy subjects and patients with cancer. Br J Clin Pharmacol 81: 1124-1133, 2016.

39. Irie T, Kuramochi M and Takahashi N: Dense accumulation of lipiodol emulsion in hepatocellular carcinoma nodule during selective balloon-occluded transarterial chemoembolization: Measurement of balloon-occluded arterial stump pressure. Cardiovasc Intervent Radiol 36: 706-713, 2013.

40. Lammer J, Malagari K, Vogl T, Pilleul F, Denys A, Watkinson A, Pitton M, Sergent G, Pfammatter T, Terraz S, et al: Prospective randomized study of doxorubicin-eluting-bead embolization in the treatment of hepatocellular carcinoma: results of the PRECISION V study. Cardiovasc Intervent Radiol 33: 41-52, 2010.

41. Kim JH, Shim JH, Lee HC, Sung KB, Ko HK, Ko GY, Gwon DI, Kim JW, Lim YS and Park SH: New intermediate-stage subclassification for patients with hepatocellular carcinoma treated with transarterial chemoembolization. Liver Int 37: 1861-1868, 2017.

42. Finn RS, Merle P, Granito A, Huang YH, Bodoky G, Pracht M, Yokosuka O, Rosmorduc O, Gerolami R, Caparello C, et al: Outcomes of sequential treatment with sorafenib followed by regorafenib for HCC: Additional analyses from the phase III RESORCE trial. J Hepatol 69: 353-358, 2018.

43. Ogasawara S, Ooka Y, Itokawa N, Inoue M, Okabe S, Seki A, Haga Y, Obu M, Atsukawa M, Itobayashi E, et al: Sequential therapy with sorafenib and regorafenib for advanced hepatocellular carcinoma: A multicenter retrospective study in Japan. Invest New Drugs 38: 172-180, 2020.

This work is licensed under a Creative Commons Attribution-NonCommercial-NoDerivatives 4.0 International (CC BY-NC-ND 4.0) License. 